\title{
Cobalt-Mediated Regioselective Synthesis of Substituted Tetrahydroquinolines ${ }^{1}$
}

Ulrich Groth,* Thomas Huhn, Christian Kesenheimer, Aris Kalogerakis

Fachbereich Chemie, Universität Konstanz, Fach M-720, Universitätsstr. 10, 78457 Konstanz, Germany

Fax +49(7531)884155; E-mail: Ulrich.Groth@uni-konstanz.de

\begin{abstract}
A regioselective synthesis of polycyclic substituted pyridines is reported. Key step is the cobalt-catalyzed intramolecular cyclization of diynenitriles, tethered by a silicon oxygen bond. Subsequent opening of the $\mathrm{Si}-\mathrm{O}$ ring led then to the related tetrahydroquinolines.
\end{abstract}

Key words: cobalt, catalysis, cycloaddition, pyridines, quinolines, ergot alkaloids

In the course of our study on transition metal-catalyzed reactions we developed a new method for the construction of linear annelated polycycles via a cobalt-mediated cyclization of silicon oxygen tethered enediynes ${ }^{2 a}$ and diynenitriles. ${ }^{2 b}$ Herein we would like to present the application of this method to the synthesis of substituted polycyclic pyridines. ${ }^{3}$ In addition to our earlier results, Malacria also reported on the chemo- and regioselective cobalt(I)-mediated cyclotrimerization of alkynes using a silylated tether. ${ }^{4}$ We decided to use for our cyclizations the commercially available $\mathrm{CpCo}(\mathrm{CO})_{2}$ and the 'Jonas catalyst' $\left[\mathrm{CpCo}\left(\mathrm{C}_{2} \mathrm{H}_{4}\right)_{2}\right],{ }^{5}$ because it promotes cyclotrimerization even at low temperature and without irradiation. ${ }^{6}$ $\mathrm{CpCo}\left(\mathrm{C}_{2} \mathrm{H}_{4}\right)_{2}$ can be easily obtained using Vollhardt's improved procedure. ${ }^{7}$ Recently the synthesis of 2 -substituted pyridines via a photocatalyzed $[2+2+2]$ cycloaddition at room temperature has been reported using $\mathrm{CpCo}(\mathrm{cod}){ }^{8}$

Our synthesis began with the deprotonation of heptynenitrile 1 with $n$-BuLi and treatment of the resulting lithiated acetylene with $\mathrm{ClMe}_{2} \mathrm{SiNEt}_{2}$. Reaction of various substituted propynylalcohols $\mathbf{3}$ with the generated silylacetylene 2 gave the diynenitriles 4 in good yields (Table 1). Cyclization of the unsubstituted diynenitrile $4 \mathbf{a}(\mathrm{R}=\mathrm{H})$ with $2.5 \% \mathrm{CpCo}(\mathrm{CO})_{2}$ in refluxing toluene with concomitant irradiation by a tungsten lamp gave the tetrahydroquinoline $\mathbf{5 a}$ in $37 \%$ yield. Use of the more effective $\mathrm{CpCo}\left(\mathrm{C}_{2} \mathrm{H}_{4}\right)_{2}$ in diethyl ether and without irradiation led to $5 \mathbf{a}$ in better yields, even at $-20{ }^{\circ} \mathrm{C}$. Similar results were obtained with the methyl-substituted $\mathbf{4 b}$ and the diynenitrile $\mathbf{4 c}$, which have been synthesized using 3-butyne-1-ol (3c, $\left.\mathrm{CH} \equiv \mathrm{CHCH}_{2} \mathrm{CH}_{2} \mathrm{OH}\right) .{ }^{9} \mathrm{Next}$, we studied the trimerization of diynenitriles with bulky substituents R: only the trimethylsilyl-substituted 3d reacted to the pyridine 5d using $\mathrm{CpCo}(\mathrm{CO})_{2}$ in refluxing toluene, but in very poor yield $(5 \%)$. In the case of $\mathrm{R}=\mathrm{Ph}, \mathrm{CH}_{2} \mathrm{OTBDMS}$ or $\mathrm{CH}_{2} \mathrm{OCH}_{3}$ no cyclization could be observed even after prolonged reaction time (Scheme 1).
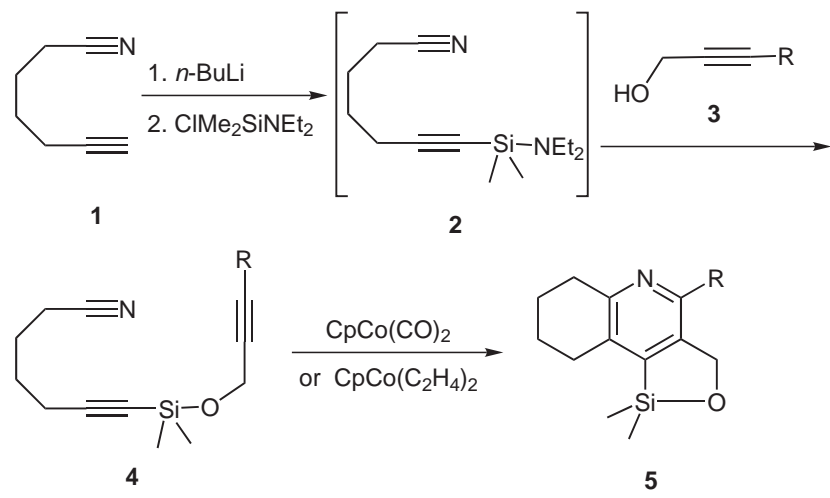

Scheme 1

Table 1 Synthesis of the Pyridines 5

\begin{tabular}{llll}
\hline Entry & 3, $\mathrm{R}$ & Yield of $\mathbf{4}(\%)$ & Yield of $\mathbf{5}(\%)$ \\
\hline 1 & 3a, $\mathrm{H}$ & 83 & $37^{\mathrm{a}}$ \\
2 & 3a, $\mathrm{H}$ & 83 & $46^{\mathrm{b}}$ \\
3 & 3a, $\mathrm{H}$ & 83 & $52^{\mathrm{c}}$ \\
4 & 3b, $\mathrm{CH}_{3}$ & 77 & $55^{\mathrm{a}}$ \\
5 & 3c, $\mathrm{H}^{\mathrm{d}}$ & 61 & $61^{\mathrm{a}}$ \\
6 & 3d, $\mathrm{Si}\left(\mathrm{CH}_{3}\right)_{3}$ & 76 & $5^{\mathrm{a}}$ \\
7 & 3d, $\mathrm{Si}\left(\mathrm{CH}_{3}\right)_{3}$ & 76 & $-^{\mathrm{c}}$ \\
\hline
\end{tabular}

a $2.5 \% \mathrm{CpCo}(\mathrm{CO})_{2}, \mathrm{hv}$, toluene, reflux, $4 \mathrm{~h}$.

b $2.5 \% \mathrm{CpCo}\left(\mathrm{C}_{2} \mathrm{H}_{4}\right)_{2}$, diethyl ether, $-20{ }^{\circ} \mathrm{C}, 18 \mathrm{~h}$.

c $2.5 \% \mathrm{CpCo}\left(\mathrm{C}_{2} \mathrm{H}_{4}\right)_{2}$, diethyl ether, $-80{ }^{\circ} \mathrm{C}$ to r.t., $18 \mathrm{~h}$.

${ }^{\mathrm{d}} \mathrm{CH} \equiv \mathrm{CHCH}_{2} \mathrm{CH}_{2} \mathrm{OH}$ was used.

On our interest for further functionalization, we found that treatment of the pyridine $\mathbf{5 a}$ with $\mathrm{H}_{2} \mathrm{O}_{2}, \mathrm{KHCO}_{3}$ and TBAF led to the disubstituted tetrahydroquinoline $\mathbf{6}$, while the same conditions without an oxidant gave the hydroxy methylene substituted tetrahydroquinoline $7 .{ }^{10}$

In order to explore the scope of the above cobalt cyclization protocol of silicon tethered precursors in natural product synthesis, the preparation of a protected indole substituted diynenitrile $\mathbf{1 2}$ was tackled to gain access to the construction of the ergot alkaloids core (Scheme 3): 


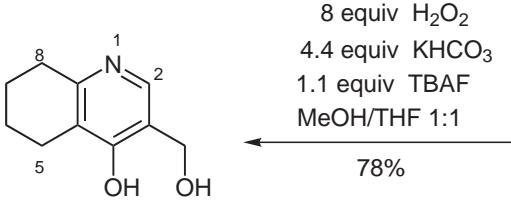

6

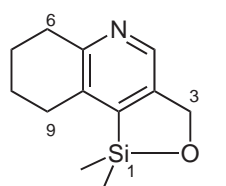

$5 a$
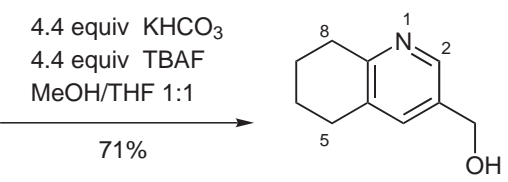

7

Scheme 2

after protection of indole 8 with $\mathrm{Si}\left[\mathrm{CH}\left(\mathrm{CH}_{3}\right)_{2}\right]_{3} \mathrm{Cl}$ (TIPSCl), introduction of trimethylsilylacetylene (TMSA) by Stevens-Castro coupling, deprotection of the resulting indole 10 with $\mathrm{K}_{2} \mathrm{CO}_{3}$ in $\mathrm{MeOH}$, treatment of the so obtained ynenitrile 11 with $n$ - BuLi/ClMe $\mathrm{SiNEt}_{2}$ and then addition of 2-propyn-1-ol led to the desired diynenitrile 12. The trimerization of $\mathbf{1 2}$ was achieved with $2.5 \%$ $\mathrm{CpCo}\left(\mathrm{C}_{2} \mathrm{H}_{4}\right)_{2}$ in diethyl ether at low temperature $\left(-80{ }^{\circ} \mathrm{C}\right.$ to r.t.) and gave the indole $\mathbf{1 3}$ in $39 \%$ yield. ${ }^{9}$ Cyclization experiments with $\mathrm{CpCo}(\mathrm{CO})_{2}$ under irradiation in refluxing toluene were not successful (obviously the indole $\mathbf{1 2}$ decomposes at high temperature). Unfortunately, the silicon-oxygen ring could not be opened using either the conditions in Scheme 2 or Vollhardt's methylationreduction protocol in the total synthesis of $( \pm)$-lysergene and $( \pm)$-LSD. ${ }^{11}$

In conclusion, the herein reported cobalt-mediated trimerization of silicon-oxygen tethered diynenitriles offers a new access toward substituted polycyclic pyridines. Application of this method has been shown in the synthesis of the indolylpyridine $\mathbf{1 3}$ with the typical $\mathrm{ABCD}$ ring annelation pattern of the ergot alkaloids. Future effort of our group is the preparation of further nitrogen containing polycycles of pharmacologically interesting natural products and related compounds by using the above-described protocol.

\section{Acknowledgment}

The authors are grateful to the Fonds der Chemischen Industrie for financial support and the WACKER Chemie GmbH for valuable starting materials.

\section{References}

(1) Transition Metal-Catalyzed Reactions in Organic Synthesis, X. For part IX, see: Fischer, S.; Groth, U.; Jung, M.; Lindenmaier, M.; Vogel, T. Tetrahedron Lett. 2005, in print.

(2) (a) Eckenberg, P.; Groth, U. Synlett 2003, 2188. (b) Eckenberg, P.; Groth, U.; Huhn, T. GIT-Fachzeitschrift für Laborwesen 1993, 892.

(3) (a) Yamazaki, H.; Wakatsuki, Y. Tetrahedron Lett. 1973, 3383. (b) Boennemann, H. Angew. Chem., Int. Ed. Engl. 1978, 17, 505; Angew. Chem. 1978, 90, 517. (c) Vollhardt, K. P. C. Angew. Chem., Int. Ed. Engl. 1984, 23, 539; Angew. Chem. 1984, 96, 525. (d) For a review see: Varela, J. A.; Saá, C. Chem. Rev. 2003, 103, 3787. (e) For the selective cyclotrimerization of two different acetylenes and a nitrile, see: Suzuki, D.; Tanaka, R.; Urabe, H.; Sato, F. J. Am. Chem. Soc. 2002, 124, 3518.

(4) Chouraqui, G.; Petit, M.; Aubert, C.; Malacria, M. Org. Lett. 2004, 6, 1519.

(5) Jonas, K.; Deffense, E.; Habermann, D. Angew. Chem., Int. Ed. Engl. 1983, 22, 716; Angew. Suppl. 1983, 1005.
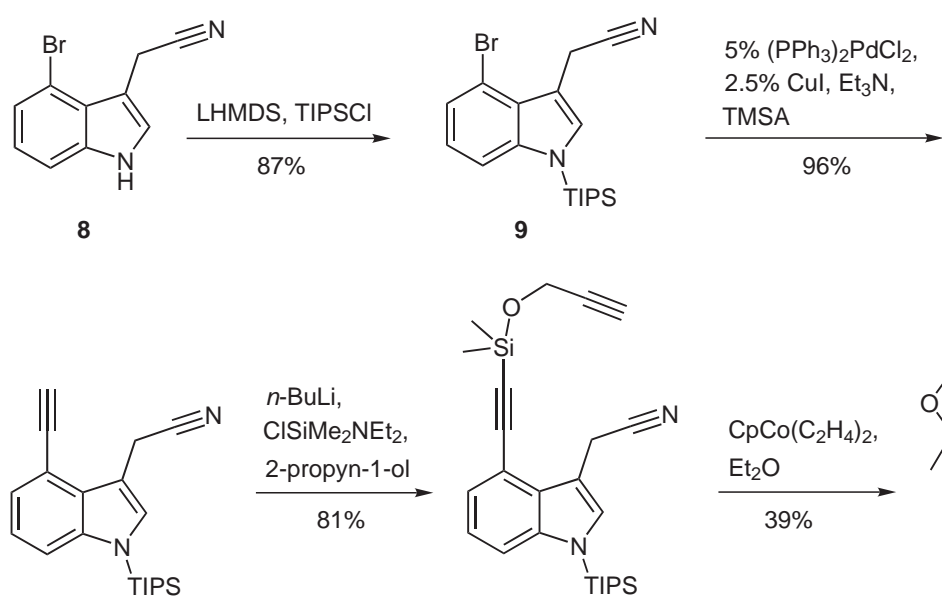

12
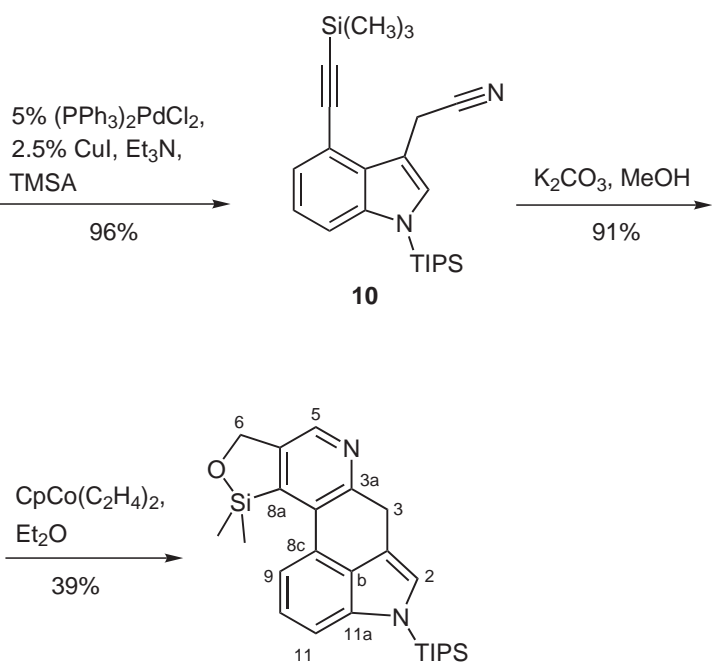

13

Scheme 3 
(6) (a) Diercks, R.; Eaton, B. E.; Guertzen, S.; Jalisatgi, S.; Matzger, A. J.; Radde, R. H.; Vollhardt, K. P. C. J. Am. Chem. Soc. 1998, 120, 8247. (b) Pelissier, H.; Rodriguez, J.; Vollhardt, K. P. C. Chem.-Eur. J. 1999, 5, 3549.

(c) Eichberg, M. J.; Dorta, R. L.; Lamottke, K.; Vollhardt, K. P. C. Org. Lett. 2000, 2, 2479. (d) Eichberg, M. J.; Dorta, R. L.; Grotjahn, D. B.; Lamottke, K.; Schmidt, M.; Vollhardt, K. P. C. J. Am. Chem. Soc. 2001, 123, 9324. (e) Dosa, P. I.; Whitener, G. D.; Vollhardt, K. P. C. Org. Lett. 2002, 4, 2075. (f) Kalogerakis, A.; Groth, U. Org. Lett. 2003, 5, 843.

(7) Cammack, J. K.; Jalisatgi, S.; Matzger, A. J.; Negrón, A.; Vollhardt, K. P. C. J. Org. Chem. 1996, 61, 4798.

(8) (a) Heller, B.; Sundermann, B.; Buschmann, H.; Drexler, H.J.; You, J.; Holzgrabe, U.; Heller, E.; Oehme, G. J. Org. Chem. 2002, 67, 4414. (b) Gutnov, A.; Heller, B.; Drexler, H.-J.; Spannenberg, A.; Sundermann, B.; Sundermann, C. Angew. Chem. Int. Ed. 2004, 43, 3795; Angew. Chem. 2004, 116, 3883.

(9) General Experimental Procedure.

a) To a solution of the diynenitrile $\mathbf{4}$ in $\mathrm{Et}_{2} \mathrm{O}$ were added 2.5 mol\% $\mathrm{CpCo}\left(\mathrm{C}_{2} \mathrm{H}_{4}\right)_{2}$ at $-80{ }^{\circ} \mathrm{C}$. The resulting mixture was warmed to r.t. under stirring over $18 \mathrm{~h}$. The organic phase was then concentrated in vacuum. Chromatography on silica gel provided pure pyridine $\mathbf{5}$.

b) A solution of $2.5 \mathrm{~mol} \% \mathrm{CpCo}(\mathrm{CO})_{2}$ in $2 \mathrm{~mL}$ toluene was added via cannula to a solution of the diynenitrile 4 in toluene and the mixture was heated at reflux under irradiation with a tungsten-lamp (Osram Vitalux $300 \mathrm{~W}$ ) for $4 \mathrm{~h}$. The organic phase was then concentrated in vacuum. Chromatography on silica gel provided pure pyridine $\mathbf{5}$. Analytical data of selected compounds.

Compound 5a: $R_{f}=0.21\left(\mathrm{Et}_{2} \mathrm{O}\right)$. $\mathrm{Mp} 44^{\circ} \mathrm{C}$. IR (film): 3010 (arom. H), 1570, $1550(\mathrm{C}=\mathrm{C}, \mathrm{C}=\mathrm{N}) \mathrm{cm}^{-1} .{ }^{1} \mathrm{H}$ NMR $(200$ $\left.\mathrm{MHz}, \mathrm{CDCl}_{3}\right): \delta=0.39\left[\mathrm{~s}, 6 \mathrm{H}, \mathrm{Si}\left(\mathrm{CH}_{3}\right)_{2}\right], 1.76-1.98(\mathrm{~m}, 4$ $\left.\mathrm{H}, \mathrm{CH}_{2}\right), 2.75(\mathrm{t}, J=11.5 \mathrm{~Hz}, 2 \mathrm{H}, \mathrm{H}-9), 2.92(\mathrm{t}, J=11.5 \mathrm{~Hz}$, $2 \mathrm{H}, \mathrm{H}-6), 5.09$ (s, $\left.2 \mathrm{H}, \mathrm{OCH}_{2}\right), 8.25$ (s, $1 \mathrm{H}$, arom. H). ${ }^{13} \mathrm{C}$ NMR $\left(50 \mathrm{MHz}, \mathrm{CDCl}_{3}\right): \delta=-0.12\left[\left(\mathrm{Si}\left(\mathrm{CH}_{3}\right)_{2}\right], 22.70\right.$, 23.00, (C-7, C-8), 29.58 (C-6), 32.08 (C-9), 69.33 (C-3), 134.49 (C-9b), 140.34 (C-4), 141.85 (C-9a), 144.72 (C-3a), $154.12(\mathrm{C}-5 \mathrm{a})$. MS (EI, $70 \mathrm{eV}): \mathrm{m} / z(\%)=219(60)\left[\mathrm{M}^{+}\right], 43$ (100) $\left[\mathrm{C}_{2} \mathrm{H}_{5} \mathrm{~N}^{+}\right]$. Anal. Calcd for $\mathrm{C}_{12} \mathrm{H}_{17} \mathrm{NOSi}$ (219.3): C, 65.71; H, 7.81. Found: C, 65.72; H, 7.79.

Compound 13: $R_{f}=0.55\left(\mathrm{Et}_{2} \mathrm{O}-\mathrm{CHCl}_{3}, 20: 1\right) . \mathrm{Mp} 89^{\circ} \mathrm{C}$. IR (film): 3060 (arom. H), $2210(\mathrm{C}-\mathrm{N}), 1600(\mathrm{C}=\mathrm{N}) \mathrm{cm}^{-1} .{ }^{1} \mathrm{H}$ $\operatorname{NMR}\left(500 \mathrm{MHz}, \mathrm{CDCl}_{3}\right): \delta=0.59\left[\mathrm{~s}, 6 \mathrm{H}, \mathrm{Si}\left(\mathrm{CH}_{3}\right)_{2}\right], 1.11$ $\left\{\mathrm{d}, J=7 \mathrm{~Hz}, 18 \mathrm{H}, \mathrm{Si}\left[\mathrm{CH}\left(\mathrm{CH}_{3}\right)_{2}\right]_{3}\right\}, 1.64\{\mathrm{sp}, J=7 \mathrm{~Hz}, 18$ $\left.\mathrm{H}, \mathrm{Si}\left[\mathrm{CH}\left(\mathrm{CH}_{3}\right)_{2}\right]_{3}\right\}, 4.56$ (s, $\left.2 \mathrm{H}, \mathrm{H}-3\right), 5.14\left(\mathrm{~s}, 2 \mathrm{H}, \mathrm{OCH}_{2}\right)$,
6.99 (s, $1 \mathrm{H}, \mathrm{H}-11$ ), 7.10 (d, J= $7 \mathrm{~Hz}, 1 \mathrm{H}, \mathrm{H}-9), 7.12$ (s, 1 $\mathrm{H}, \mathrm{H}-2), 7.30\left(\mathrm{dd}, J_{1}=J_{2}=7 \mathrm{~Hz}, 1 \mathrm{H}, \mathrm{H}-10\right), 8.20(\mathrm{~s}, 1 \mathrm{H}$, $\mathrm{H}-5) .{ }^{13} \mathrm{C} \mathrm{NMR}\left(125 \mathrm{MHz}, \mathrm{CDCl}_{3}\right): \delta=0.63\left[\mathrm{Si}\left(\mathrm{CH}_{3}\right)_{2}\right]$, $12.67\left\{\mathrm{Si}\left[\mathrm{CH}\left(\mathrm{CH}_{3}\right)_{2}\right]_{3}\right), 18.06\left\{\mathrm{Si}\left[\mathrm{CH}\left(\mathrm{CH}_{3}\right)_{2}\right]_{3}\right\}, 31.78(\mathrm{C}-$ 3), $68.94\left(\mathrm{OCH}_{2}\right), 112.88(\mathrm{C}-2 \mathrm{a}), 114.07,114.13,122.40$ (C9, C-10, C-11), 124.98 (C-2), 126.94 (C-8c), 129.68 (C11b), 132.20 (C-8b), 139.39 (C-11a), 140.27 (C-8a), 141.35 (C-5), 143.15 (C-5a), 154.06 (C-3a). MS (EI, $70 \mathrm{eV})$ : $m / z(\%)=448(100)\left[\mathrm{M}^{+}\right], 405(55)\left[\mathrm{M}^{+}-\mathrm{C}_{3} \mathrm{H}_{7}\right]$. Anal. Calcd for $\mathrm{C}_{26} \mathrm{H}_{36} \mathrm{~N}_{2} \mathrm{OSi}_{2}$ (448.8): C, 69.59; H, 8.09. Found: C, 69.48; H, 7.96.

(10) (a) Tamao, K.; Yoshida, J.-L.; Takahashi, M.; Yamamoto, H.; Kakui, T.; Matsumoto, H.; Kurita, A.; Kumada, M. J. Am. Chem. Soc. 1978, 100, 290. (b) Tamao, K.; Kakui, T.; Kumada, M. J. Am. Chem. Soc. 1978, 100, 2268.

(c) Tamao, K.; Ishida, N.; Tanaka, T.; Kumada, M. Organometallics 1983, 2, 1694. (d) Tamao, K.; Maeda, K.; Yamaguchi, T.; Ito, Y. J. Am. Chem. Soc. 1989, 3984. (e) Tamao, K.; Hayashi, T.; Ito, Y. In Frontiers of Organosilicon Chemistry; Bassindale, A. R.; Gaspar, P. P., Eds.; Royal Society of Chemistry: Cambridge, 1991, 197 Analytical data of the desilylated compounds.

Compound 6: $R_{f}=0.29\left(\mathrm{MeOH}-\mathrm{CHCl}_{3}, 1: 10\right) . \mathrm{Mp} 189^{\circ} \mathrm{C}$. IR (film): $3350(\mathrm{OH}), 1620(\mathrm{C}=\mathrm{O}) \mathrm{cm}^{-1}$. ${ }^{1} \mathrm{H}$ NMR (200 $\left.\mathrm{MHz}, \mathrm{CDCl}_{3}\right): \delta=1.52-1.74(\mathrm{~m}, 4 \mathrm{H}, \mathrm{H}-6, \mathrm{H}-7), 2.33$ (t, 2 $\mathrm{H}, \mathrm{H}-8), 2.48(\mathrm{t}, 2 \mathrm{H}, \mathrm{H}-5), 4.24(\mathrm{~s}, 2 \mathrm{H}, \mathrm{OH}), 4.36(\mathrm{~s}, 2 \mathrm{H}$, $\left.\mathrm{OCH}_{2}\right), 7.34$ (s, $1 \mathrm{H}$, arom. H). ${ }^{13} \mathrm{C}$ NMR $\left(50 \mathrm{MHz}, \mathrm{CDCl}_{3}\right)$ : $\delta=21.68,22.03(\mathrm{C}-6, \mathrm{C}-7), 27.02(\mathrm{C}-5), 35.32(\mathrm{C}-8), 60.55$ (C-OH), 123.52 (C-3), 124.23 (C-4a), 133.50 (C-2), 145.93 (C-8a), 178.43 (C-4). MS (EI, $70 \mathrm{eV}): m / z(\%)=179(90)$ $\left[\mathrm{M}^{+}\right], 161(100)\left[\mathrm{M}^{+}-\mathrm{H}_{2} \mathrm{O}\right]$. Anal. Calcd for $\mathrm{C}_{10} \mathrm{H}_{13} \mathrm{NO}_{2}$ (178.2): C, 67.40; H, 6.79. Found: C, 67.36; H, 6.88. Compound 7: $R_{f}=0.07\left(\mathrm{Et}_{2} \mathrm{O}\right) . \mathrm{Mp} 49^{\circ} \mathrm{C}$. IR (film): 3300 $(\mathrm{OH}), 1565(\mathrm{C}=\mathrm{C}) \mathrm{cm}^{-1} .{ }^{1} \mathrm{H}$ NMR $\left(200 \mathrm{MHz}, \mathrm{CDCl}_{3}\right)$ : $\delta=1.62-1.89\left(\mathrm{~m}, 4 \mathrm{H}, \mathrm{CH}_{2}\right), 2.64$ and $2.75(2 \times \mathrm{t}, J=11 \mathrm{~Hz}$, $4 \mathrm{H}, \mathrm{H}-5, \mathrm{H}-8), 4.07$ (s, $2 \mathrm{H}, \mathrm{OH}), 4.51\left(\mathrm{~s}, 2 \mathrm{H}, \mathrm{OCH}_{2}\right), 7.30$ and $8.11\left(2 \times \mathrm{s}, 2 \mathrm{H}\right.$, arom. H). ${ }^{13} \mathrm{C} \mathrm{NMR}\left(50 \mathrm{MHz}, \mathrm{CDCl}_{3}\right)$ : $\delta=21.59,21.96$ (C-6, C-7), 27.65 (C-8), 30.76 (C-5), 60.85 (C-OH), 131.23, 133.26 (C-3, C-4a), 135.13 (C-4), 144.07 $(\mathrm{C}-2), 155.02$ (C-8a). MS (EI, $70 \mathrm{eV}): \mathrm{m} / z(\%)=163(100)$ $\left[\mathrm{M}^{+}\right], 134(80)\left[\mathrm{M}^{+}-\mathrm{CHO}\right]$. Anal. Calcd for $\mathrm{C}_{10} \mathrm{H}_{13} \mathrm{NO}$ (163.2): C, 73.59; H, 8.03. Found: C, 73.62; H, 8.11.

(11) (a) Saá, C.; Crotts, D. D.; Hsu, G.; Vollhardt, K. P. C. Synlett 1994, 487. For the recently synthesis of the ergot alkaloids core and related compounds see: (b) Hendrickson, J. B.; Wang, J. Org. Lett. 2004, 6, 3. (c) Kalinin, A. V.; Chauder, B. A.; Rakhit, S.; Snieckus, V. Org. Lett. 2003, 5, 3519. 